\title{
Review Paper \\ Improve Health of the Elderly People With M-Health and Technology
}

\author{
Reza Safdari ${ }^{1} \oplus$, Ahmad Reza Shams Abadi ${ }^{1},{ }^{*}$ Shahrbanoo Pahlevany Nejad ${ }^{1} \odot$
}

1. Department of Health Information Management, School of Allied Medical Sciences, Tehran University of Medical Sciences, Tehran, Iran.

\begin{tabular}{l|l}
$\begin{array}{l}\text { Use your device to scan } \\
\text { and read the article online }\end{array}$ \\
Cftation: Safdari R, Shams Abadi AR, Pahlevany Nejad Sh. [Improve Health of the Elderly People With M-Health and Tech- \\
nology (Persian)]. Iranian Journal of Ageing. 2018; 13(3):288-299. https://doi.org/10.32598/SIJA.13.3.288
\end{tabular}

(c) (1) (8)

Funding: See Page 296

Received: 15 Apr 2018 Accepted: 20 Jul 2018

Keywords M-health, Elderly, Mobile health devices, Health care, Technology

\section{ABSTRACT}

Objectives Advanced educational techniques with smartphones help medical staff to control diseases and reduce their complications. Application of communication technologies in health services through changing patients' behaviors (especially self-care) has had a growing trend in the prevention, treatment, and medical education of different age groups, especially the elderly. The support role of families for the elderly (despite their rising life expectancy) is decreasing mainly due to the generation gap between youth and the elderly, lower number of children, and higher life expectancy of the elderly. The purpose of this paper is to assess and improve the health of the elderly people with $\mathrm{m}$-health (use of mobile telecommunications and wireless multimedia to integrate and develop successful health care delivery systems) and technology.

Methods \& Materials The present study is a review study conducted through the search of reputable scientific resources such as Scopus, ScienceDirect, Cochrane, SID, Elsevier, ProQuest as well as advanced search on Google and Google Scholar in 2010-2017. It should be noted that a number of papers used in this study have been systematically reviewed, which would contribute to the comprehensiveness of the research result. $A$ total of 65 papers and research papers were reviewed, of which 31 were used in this study.

Results Findings indicate that the main challenge in using $\mathrm{m}$-health in the elderly is the lack of electronic literacy and phobia of technology. Resolving this problem requires the provision of infrastructures, codes and standards and more research in this area. Cellular technology (what mobile phone networks are based on) can play a key role in controlling the health of older diabetic patients (as an example) and using these technologies in Iran and other countries with acceptable justification can be used as a complementary tool in modern medicine.

Conclusion Mobile care not only is effective in reducing costs by providing easy access to care and its various aspects, it also improves patient's relationships and care providers as well as increasing self-care effectiveness, especially in old age. Recent advances in mobile technology provide many opportunities for improving the health and well-being of individuals; for example, ability to monitor and rapidly report changes in their health status, education of a healthy lifestyle, quick diagnosis, facilitation in information exchange, and promotion of health care. This technology can be effective in the sense of autonomy and safety of the elderly and it can improve their quality of life. Also, the use of this tool can increase the satisfaction of the elderly and encourage them to continue their treatment and eventually prolong their lives.

\footnotetext{
* Corresponding Author:
}

\section{Shahrbanoo Pahlevany Nejad, MSc. Student}

Address: Department of Health Information Management, School of Allied Medical Sciences, Tehran University of Medical Sciences, Tehran, Iran. Tel: +98 (919) 3345676

E-mail: shpahlevany@gmail.com 


\section{Extended Abstract}

\section{Objectives}

$\mathbf{M}$

odern educational methods using smartphones can help control illnesses and reduce their complications [1]. Application of communication technologies in the field of health services and its proper management by changing people's attitudes and, in particular, self-care, can have a significant effect on the prevention, treatment and medical education of people, especially the elderly [2]. The supporting role of families is declining mainly due to an increase in the gap between younger and older generations, reduction in the number of children, and increase in the elderly's life span. This situation will increase the need for more healthcare services during the aging period and providing the necessary prerequisites where health information technology is one of the most important components [3]. The purpose of this article is to examine the role and importance of mobile health technology in promoting community health, with a focus on the health of the elderly.

\section{Methods and Materials}

The present study is a review study by searching for keywords related to the comparative category of mobile applications in the elderly such as "mobile health," "aging," "health promotion," "technology and health care" in reputable scientific resources such as ProQuest, Scopus, ScienceDirect, Cochrane, Iranian Scientific Information Database (SID), Elsevier as well as an advanced search in Google and papers published in Google Scholar from 2010 to 2017.

This research has been approved by the Faculty of Paramedical Sciences at Tehran University of Medical Sciences. A number of the papers used in this study are systematic reviews that will help us comprehend the results of the study. A total of 65 research papers (2 from SID, 2 from ScienceDirect, 24 from Scopus, 3 from ProQuest, 15 from Elsevier, 5 from Cochrane, and 14 from other databases) were examined. Of these, 29 most relevant papers were selected and used in the study. In a similar study in Sahara, Africa, 475 papers were systematically reviewed where PubMed, EMBASE, Web of Science, Cochrane and some other databases from 1992 to 2012 were reviewed. It was suggested that most problems in previous studies were related to the short period of review and the lack of enough follow-up period.

\section{Result}

Findings indicate that the main challenge in using mobile health in the elderly is lack of electronic literacy and resistance to use technology [4]. This requires the provision of infrastructures, regulations and standards, and more research in this field [5]. Mobile health technology can play a key role in controlling the health of older diabetic patients $[6,7]$. The use of these technologies in Iran and other countries has drawn considerable attention and can be used as a complementary tool in modern medicine in the future [8].

In conducted studies, the use of mobile health technology to diagnose diseases has been reported as a component that can accelerate the diagnosis and monitoring the symptoms of disease remotely [5]. The use of portable tools for the transmission of educational concepts in both developed and developing worlds is increasing, especially when combined with audio, video, and multimedia [9]. Patients can receive care through the use of this technology anywhere even at home through ease-of-use applications, and mobile-based technologies in resource-limited settings can result in improved care outcomes [10].

This technology can bridge the gap between the demand for information and its accessibility, but this requires an increase in awareness and skill in the use of electronic information and communication resources such as mobile health [11]. In literature, the existing challenges to the implementation of mobile health are as follows: absence of a predictive system in some of applications, inconsistency in the differential diagnosis of diseases, lack of connection and linkage to health systems, lack of appropriate care and follow-up systems, and poor quality of provided cares.

Also, the effects of mobile health have not been welldocumented in some of these studies [12]. The findings show that sending text messages to patients with type 2 diabetes leads to self-care behaviors such as adherence to proper diet and medication, self-monitoring of blood glucose levels, daily exercise, and foot self-care [13].

\section{Conclusion}

The use of mobile phones in providing care not only helps in reducing costs and causes easy access to various healthcare services, but also improves relations between patients and caregivers and increases the impact of self-care especially for older adults [11]. Recent advances in mobile technology provide many oppor- 
tunities for improving the health and well-being of individuals, the ability to rapidly monitor and report the changes in health, helping to create a healthy lifestyle, quick diagnosis of health conditions, facilitating information, and promotion of health care [14].

This technology can help the independence of the elderly and improve their quality of life [15]. Also, the use of this technology can increase the satisfaction of the elderly and motivate them to continue their treatment and, finally, to survive [16]. However, security and privacy concerns still exist [17], and legal constraints remain a barrier to the development of this technology; and also there is resistance to the use of this technology [18]. There is no adequate knowledge of this technology, and local standards have not been specifically defined in this regard.

\section{Ethical Considerations}

\section{Compliance with ethical guidelines}

In conducting this research, there was no need to follow the ethical guidelines.

\section{Funding}

This research did not receive any specific grant from funding agencies in the public, commercial, or not-for-profit sectors.

\section{Conflict of interest}

The authors declared no conflict of interest. 


\title{
ارتقاى سلامت سالمندان جامعه مبتنى بر سلامت همراه و تكنولورى
}

\author{
رضا صفدرى' اه، احمدرضا شمس آبادى'، "تشهربانو يهلوانىنزاد'ه
}

1- كروه مديريت اطلاعات سلامت، دانشكده ييرائشكى، دانشكاه علوم يزٔشكى وخدمات بهداشتى درمانى تهران، تهران، ايران.

\begin{abstract}
حكنי

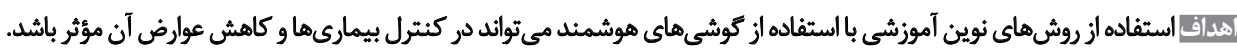

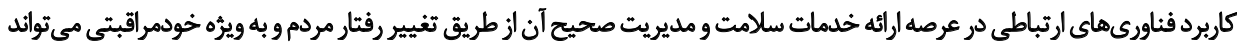

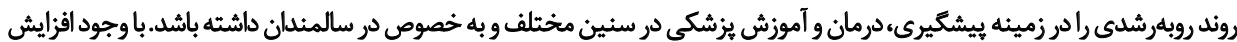

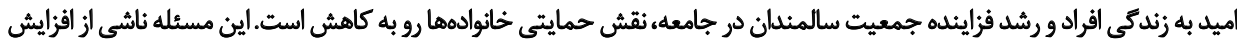

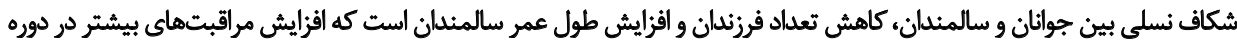

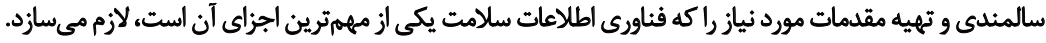

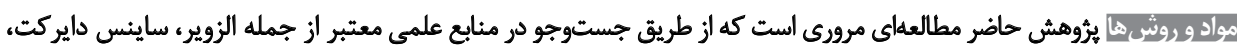

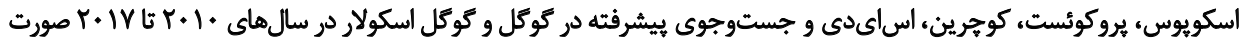

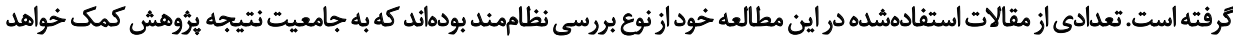

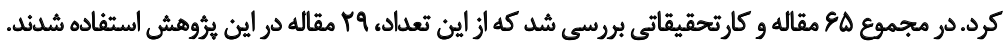

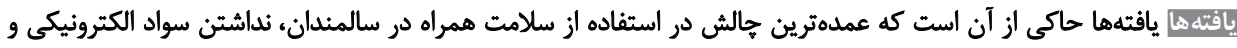

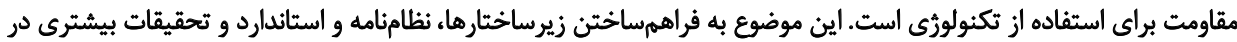

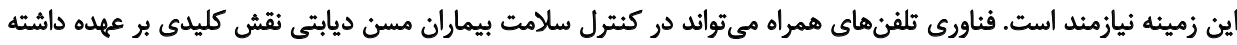

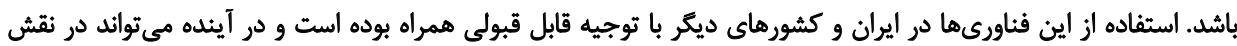
ابزار مكمل در يزٔشكى نوين استفاده شود.

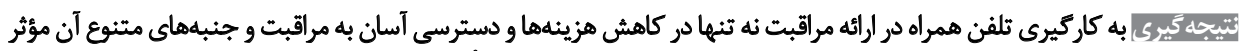

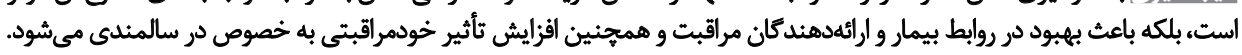

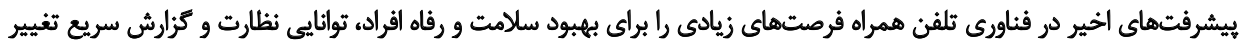

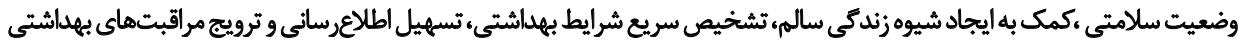

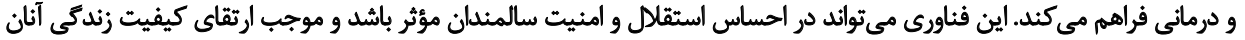

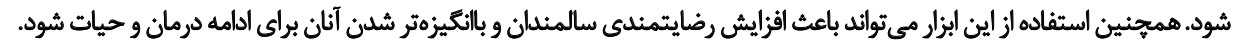

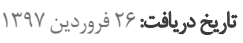

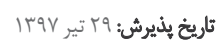

\section{كليدواروها: \\ سيلامت همراه،

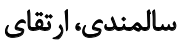

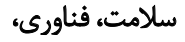 مراقبتهاى سلامت فياري}

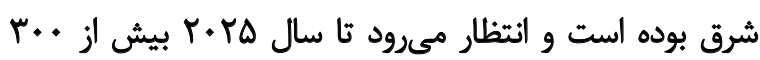

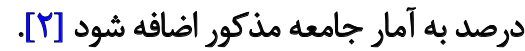

dates

سلامت همراه' اصطلاحى است كه براي برنامههاي كاربردى

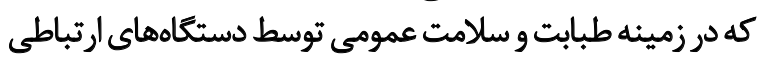

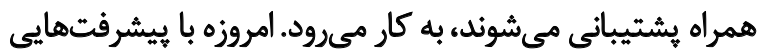

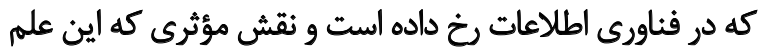

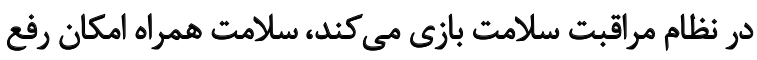

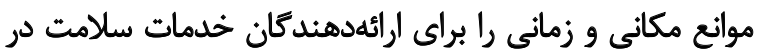

1. Mobile health ( $m$-health)
سالمندى يكى از دورانهاى حساس زندكى است كه فرد

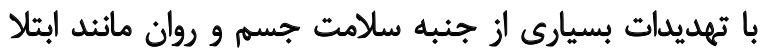

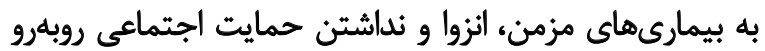

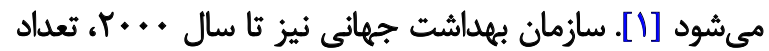

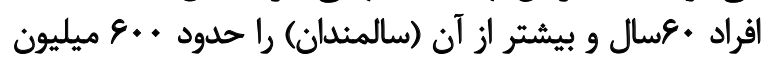

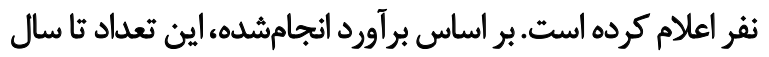

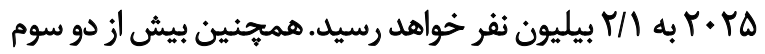

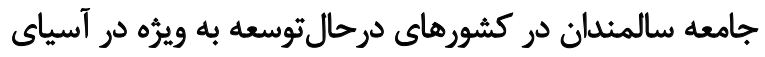

\section{ㄷ.}


متناسب با وضعيت سالمندان ساخته شدهاند، كمك بزركى براى براي

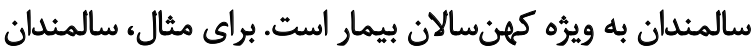

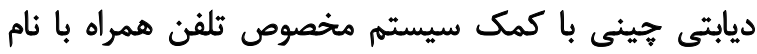

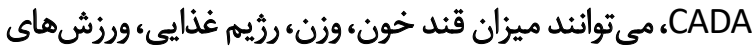

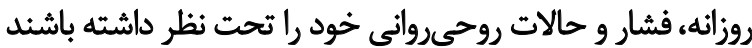

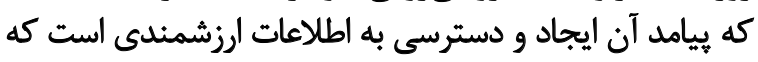

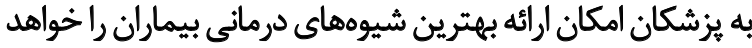

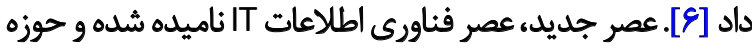

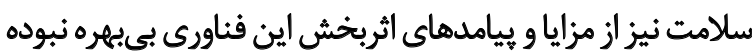

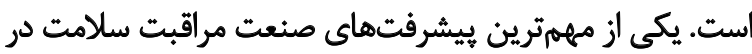

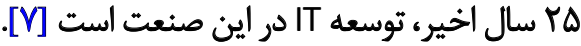
جنانجِه دوره سالمندى با سلامت و رفاه همراه شود، دوران

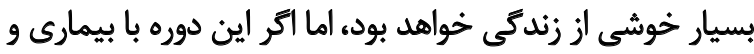

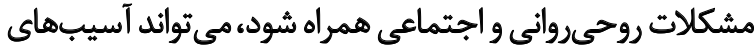

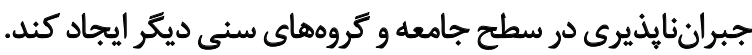

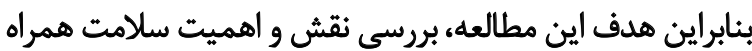

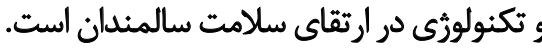

$$
\text { روش مطالعه }
$$

هروهش حاضر نوعى مطالعه مرورى است كه از طريق

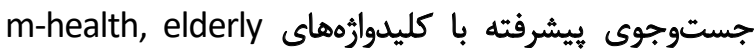
mobile health devices, health care and technology و معادل فارسى آنها با عناوين سلامت همراه، مراقبت سلامت

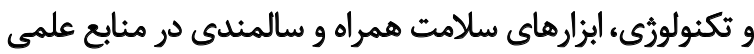

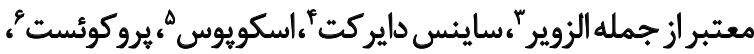

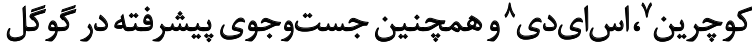

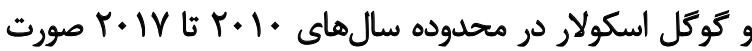

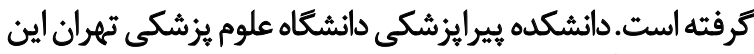

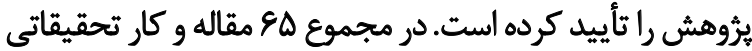

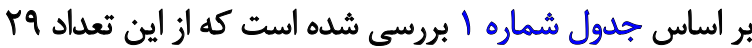

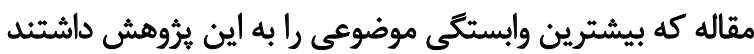

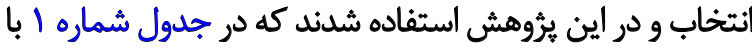
3. Elsevier
4. Science direct
5. Scopus
6. Pro Quest
7. Cochrane
8. SID

$$
\text { راستاي ارائه خدمات باكيفيت رفع كرده است [1]]. }
$$

همجنين دستكاههاى موبايل كه دائم به منابع اطلاعاتى متصل

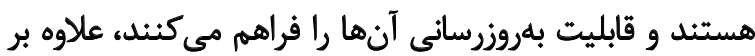

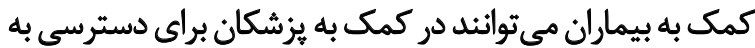

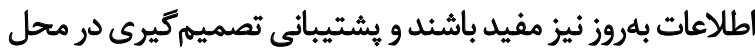

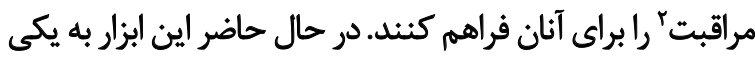

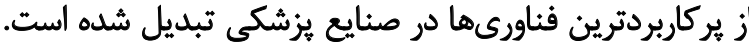

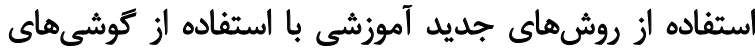

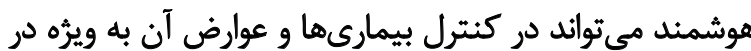

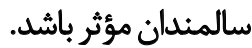

سلامت همراه يكى از كليدىترين مؤلفههاى نظام ملى سلامت

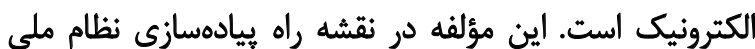

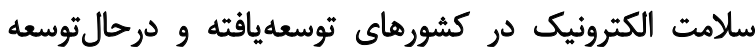

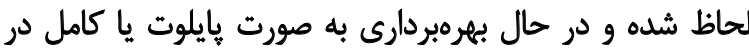

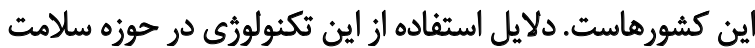

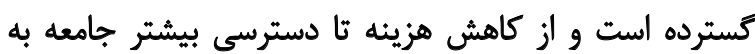

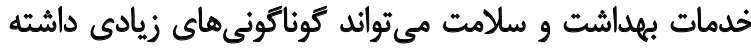

باشد [ب].

سازمان بهداشت جهانى سالمندان را نيروى جديد براى توسعه

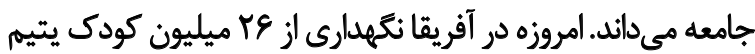

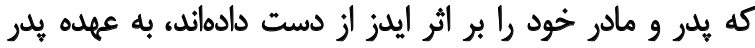

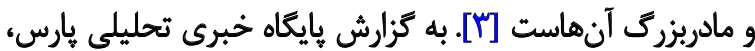

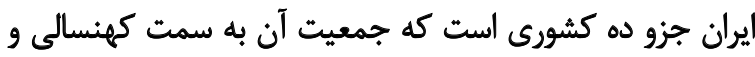

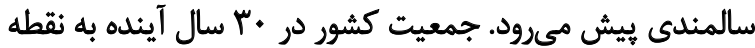

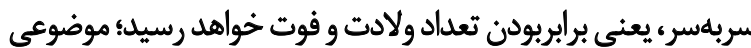

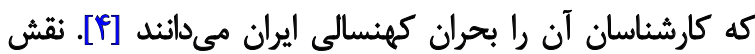

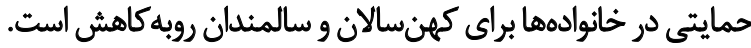

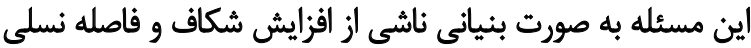

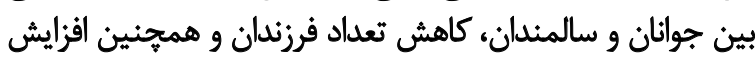

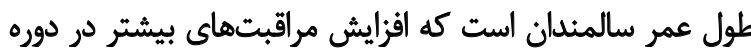

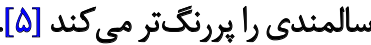

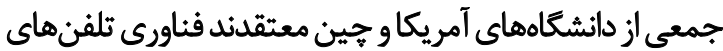

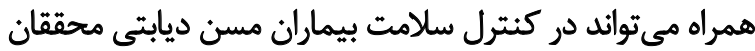

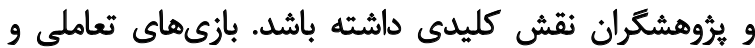

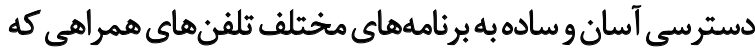

2. Point of Care (POC)

\begin{tabular}{|c|c|c|c|c|c|c|}
\hline الب آى & ساينسدايركت & السكويوس & بروكونست & كوهرين & الزوير & ديكّر سايتها \\
\hline$r$ & $r$ & $M$ & $r$ & $\Delta$ & 10 & if \\
\hline
\end{tabular}

جدول ا. تعداد مقالات بررسى شده بر حسب بإيكاه جستوجوشده

r 
و مكانى كه نياز باشد در اختيار متخصصان قرار دهد، بدون اينكه

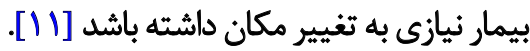

تيروموتى "و همكاران در مطالعهاى كه براى تعيين كاربرد

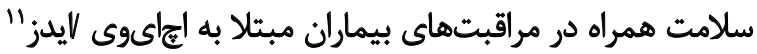

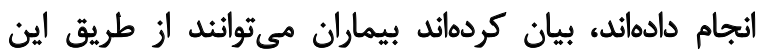

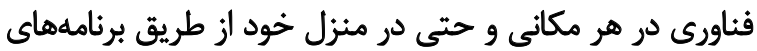

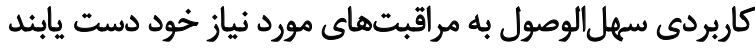

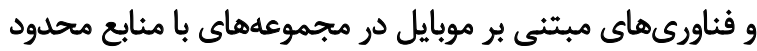

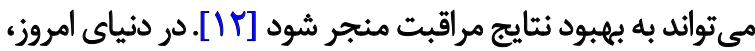

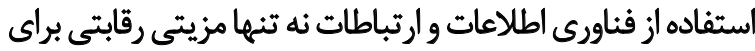

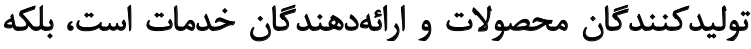

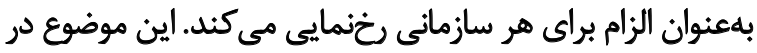

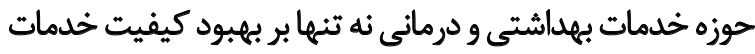

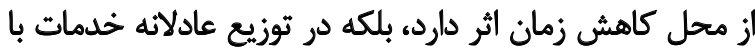

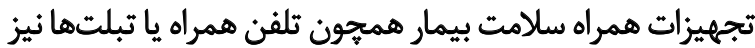

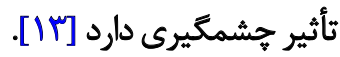

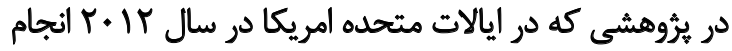

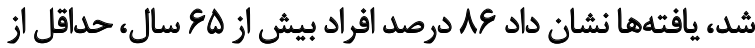

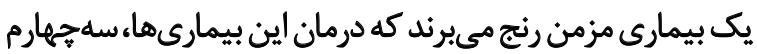

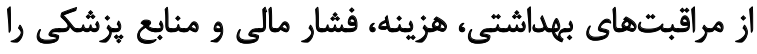

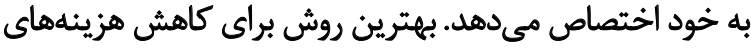

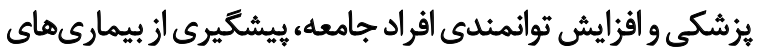

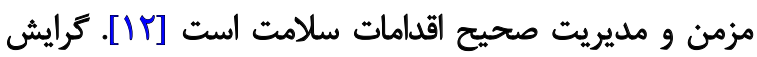

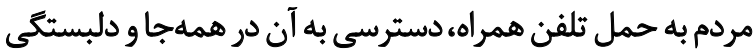

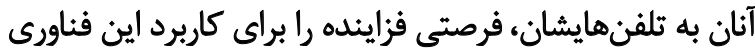

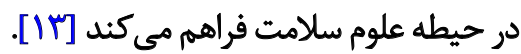

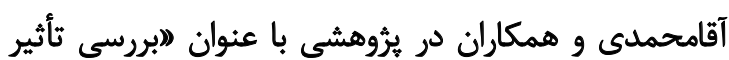

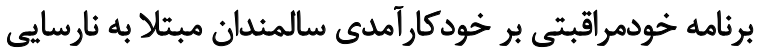

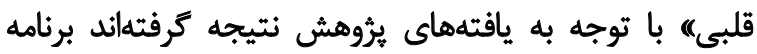

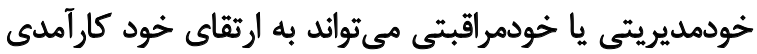

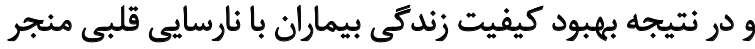

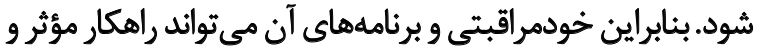

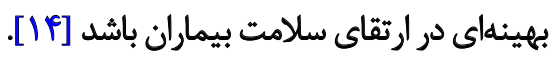

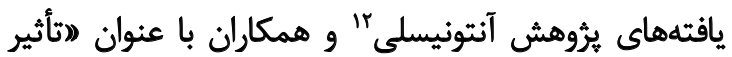

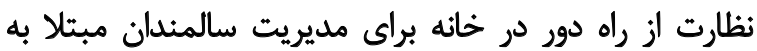

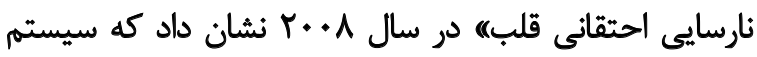

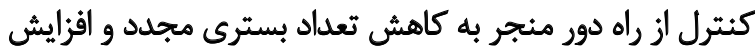

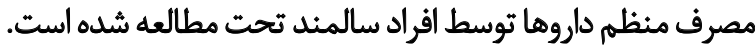
[1ه]]. عجمى و همكاران در مقاله فناورى اطلاعات درات در فرايند

10. Thiromurty

11. HIV/AIDS

12. Antonicelli
توجه به نوع يايعاه اطلاعاتي دستهبندى شدهاند.

ياقتهها

شيوع روبهرشد بيمارىهاى غيرواكير مانند ديابت و وانيت

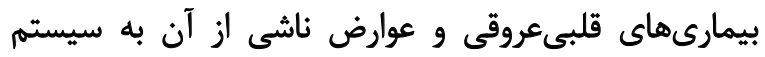

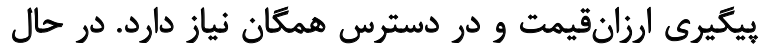

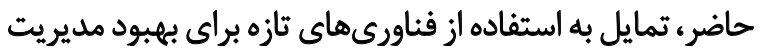

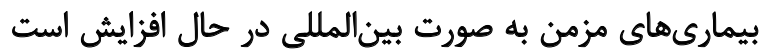

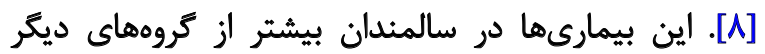

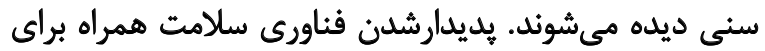

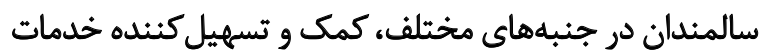

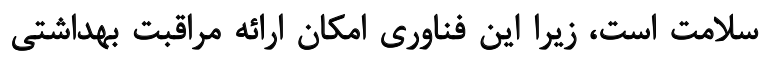

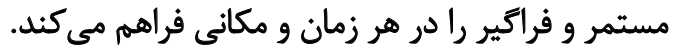

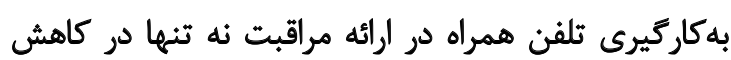

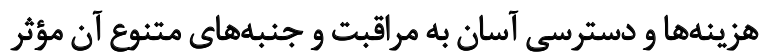

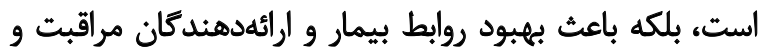

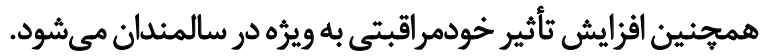

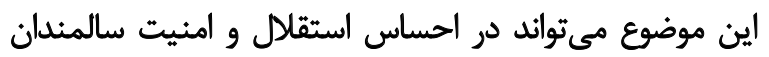

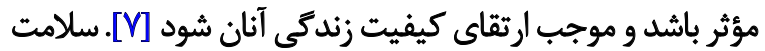

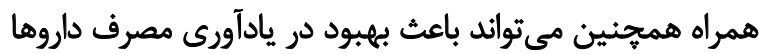

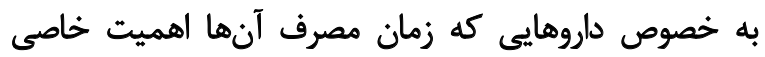

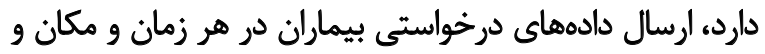

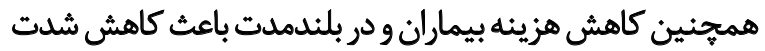

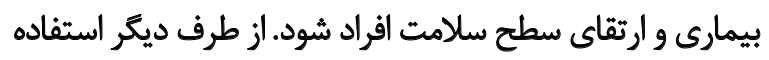

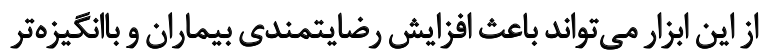

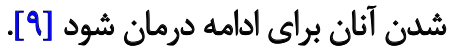

در يُروهش فروتن و همكاران، فناورى سلامت همراه يكى از براز

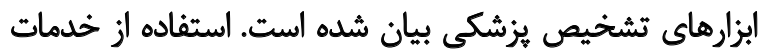

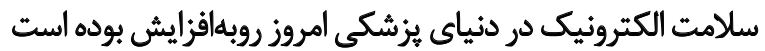

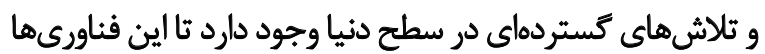

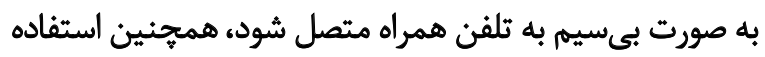

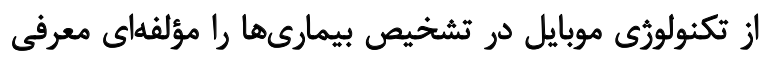

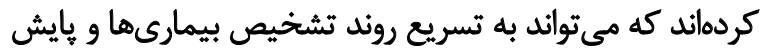

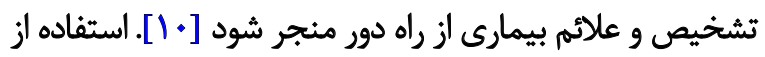

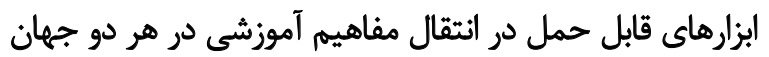

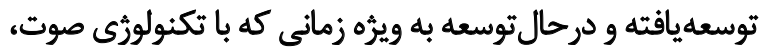

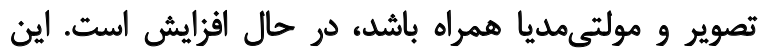

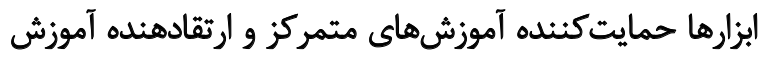

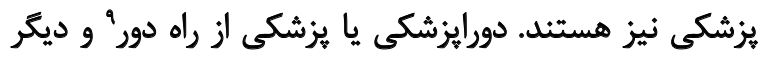

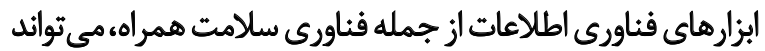

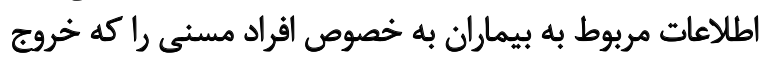

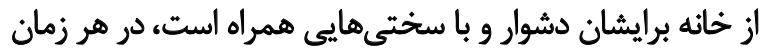

9. Telemedicine 
براى آموزش، لمدرصد براى توصيههاى غذايى، هدرصد براى

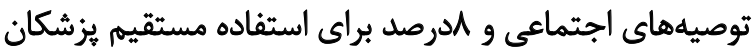

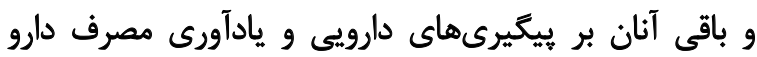

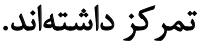

اكثر برنامههاي كاربردى همراه از نظر آنان قابليت اتصال

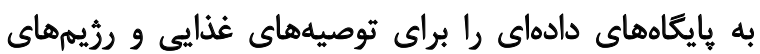

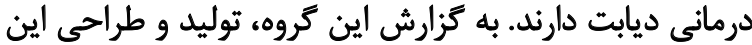

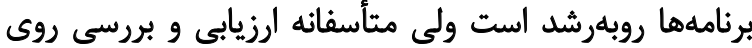

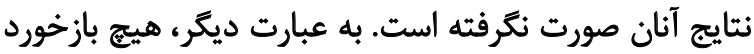

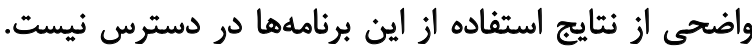

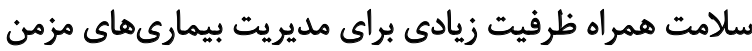

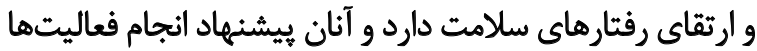

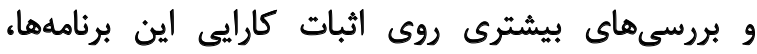

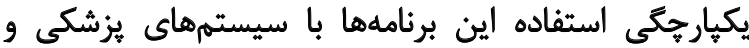

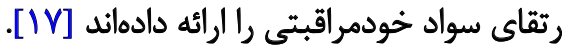

كاظمى و همكاران در مقالداى با عنوان (انقش شبكدهاي

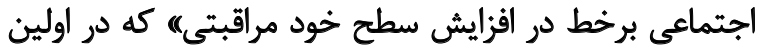

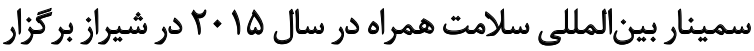

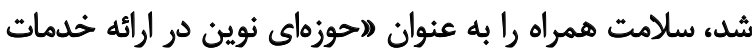

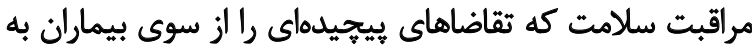

عنوان مشتريان سلامت ايجاد مي كندها تعريف كردهاند [1/]]. در بررسى ديكر كه يوسف كاندايور" و و همكاران در سال

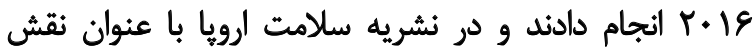

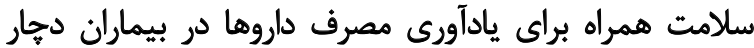

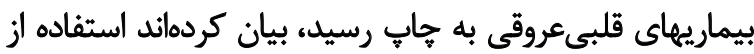

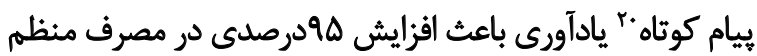

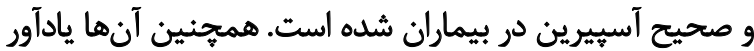

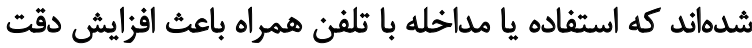

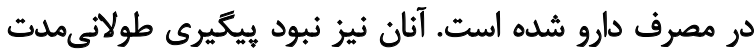

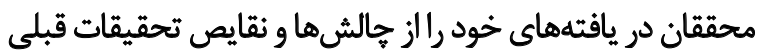

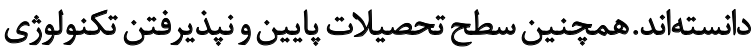

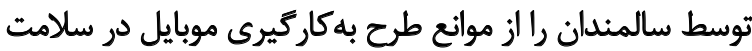

سالمندان دانستهاند [19].

در يُؤوهش تأثير آموزش به كمك ثلفن همراه بر كاهش

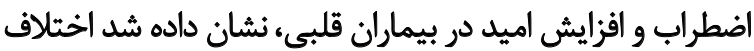

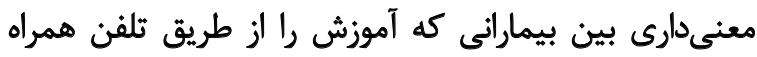

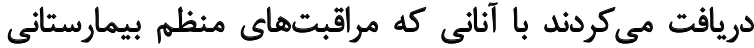

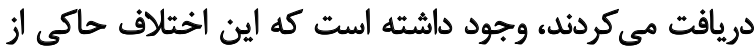

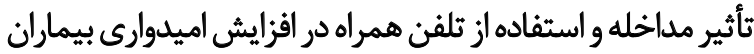

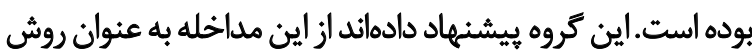

19. Gandapur

20. SMS
رائه خدمات درمانى توسط يزشكان، فناورى اطلاعات را راهي

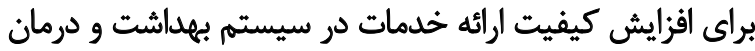

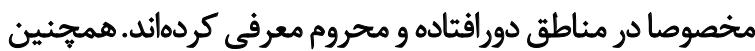

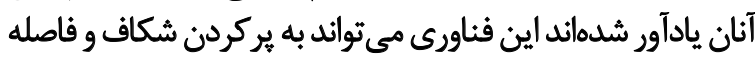

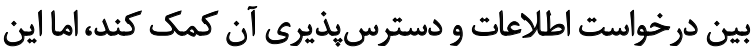

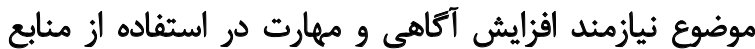

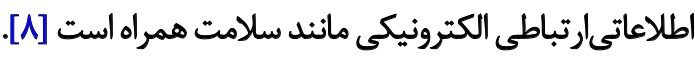

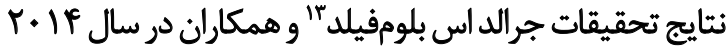

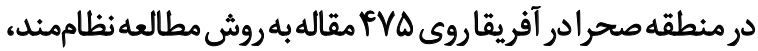

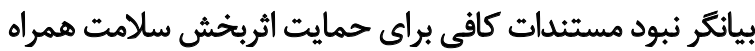

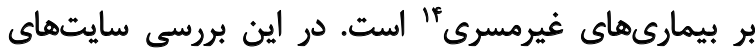

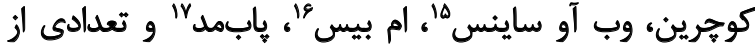

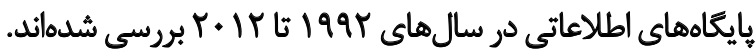

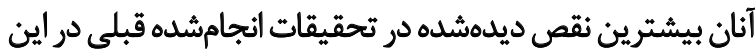

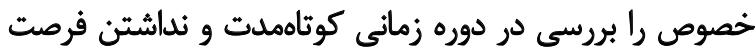

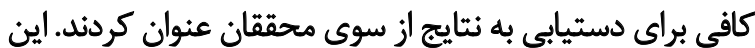

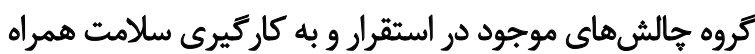

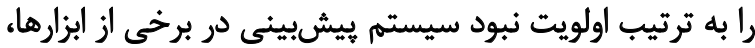

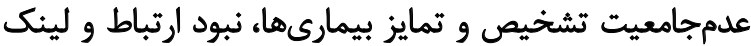

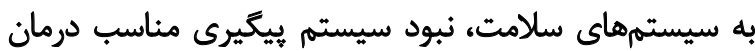

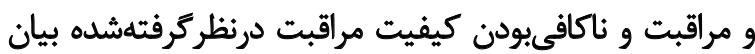

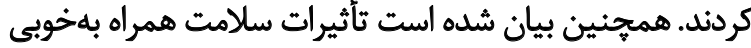

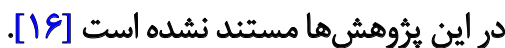

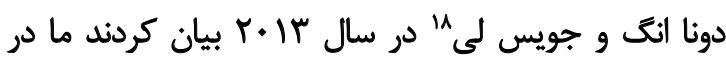

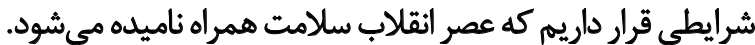

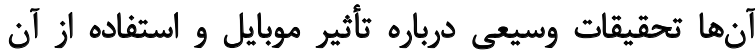

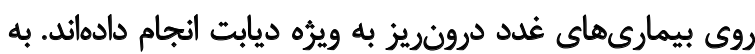

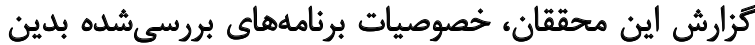

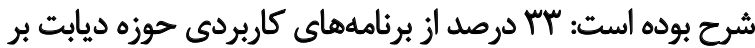

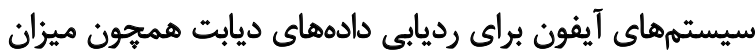

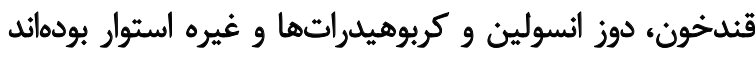

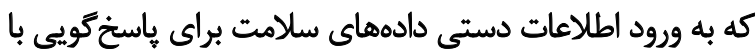

كمك سيستم نيازمند بودند [ [IV]

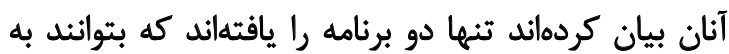

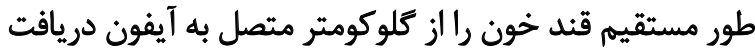

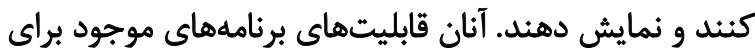

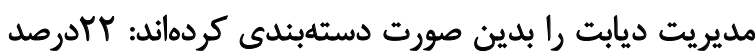

13. Gerald S Bloomfield

14. Non Communicable Disease (NCD)

15. Web of science

16. $M$ base

17. Pub med

18. Donna Eng \& Joyce Lee 
كاهش هزينههاى تحميل شده به بيماران شود. تكنولوريى سلامت

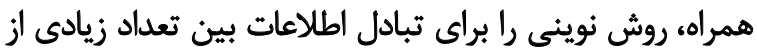

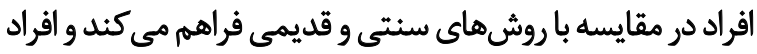

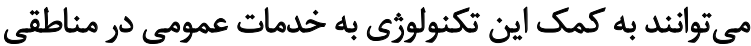

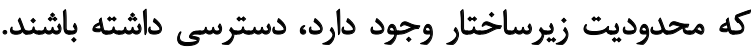

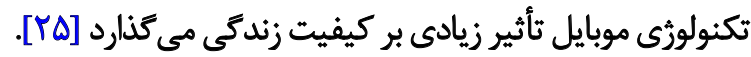
ثيروهشكران معتقدند فناورى تلفنهاى همراه ميتواند دري

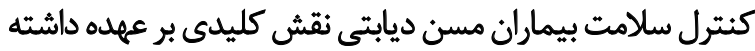

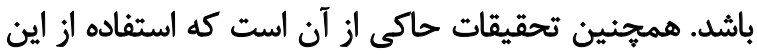

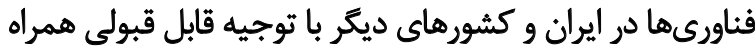

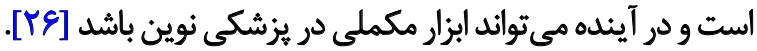

\section{نتيجه كيرى نمبايى}

جالشها و و مشكلات بياده سازى فناورى سلامت همراه

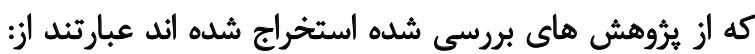

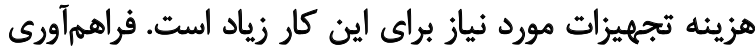

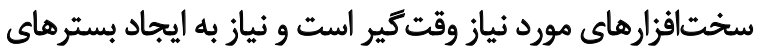

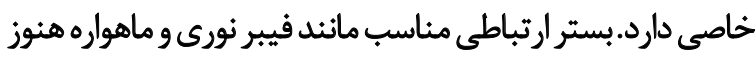

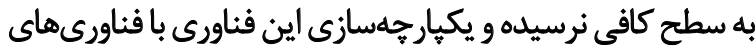

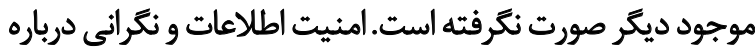

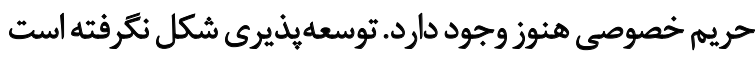

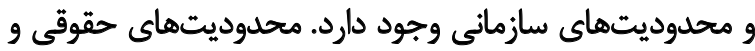

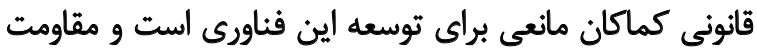

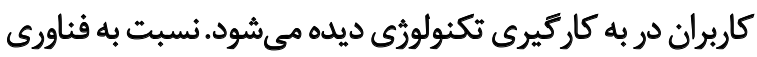

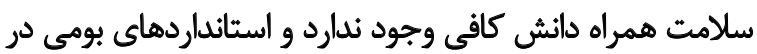
اين خصوص تعريف نشدهاند [TV]

به كزارش تروه دانستنىهاى خبر گزارى فارس، مشكل اصلى

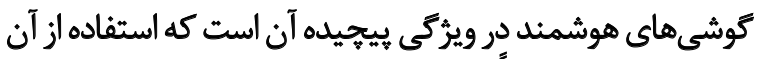

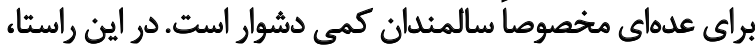

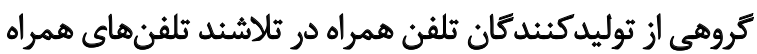

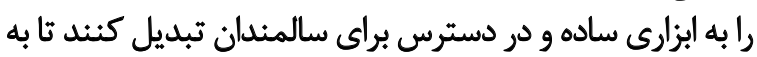

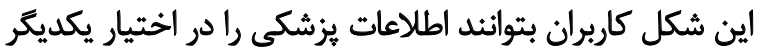

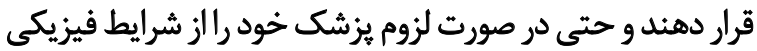

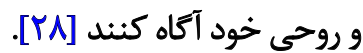

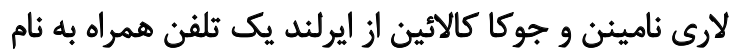

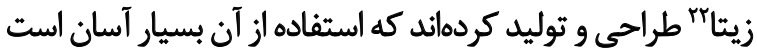

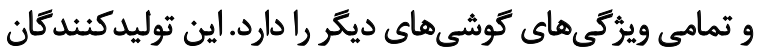

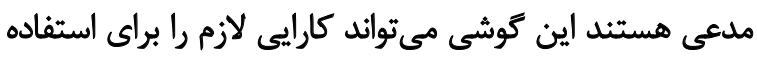

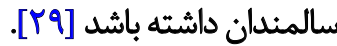

شبكههاى تلفن همراه يا سلامت همراه بيشتر از هر سيستم

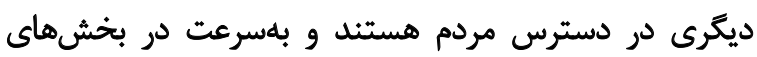

تكميلى براى بيكيرى وضعيت بيماران قلبى استفاده شود [9 19].

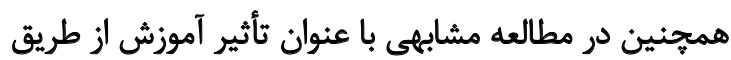

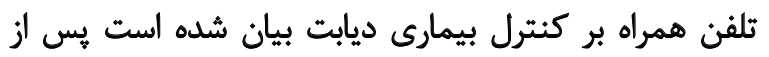

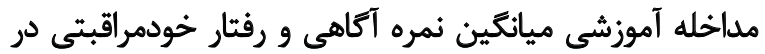

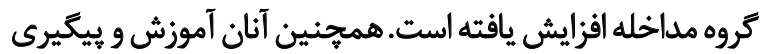

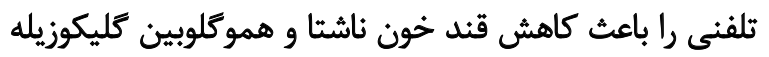

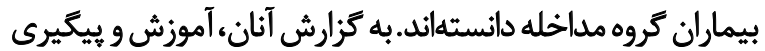

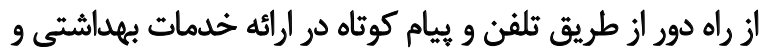

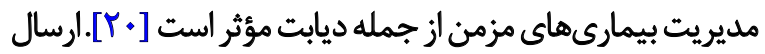

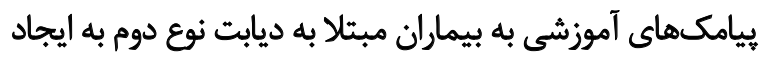

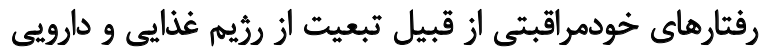

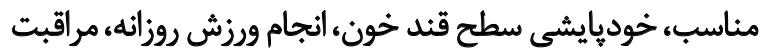

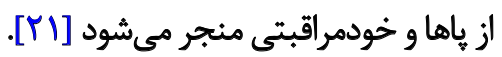

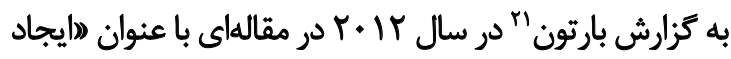

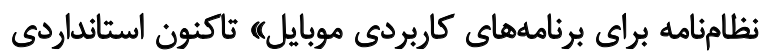

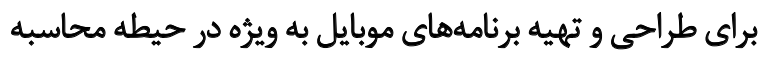

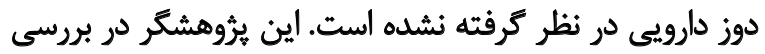

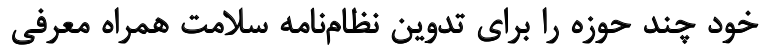

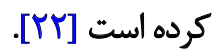

بحث

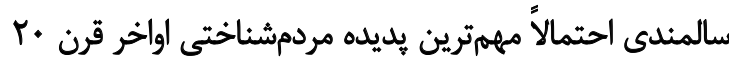

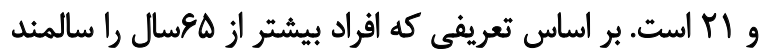

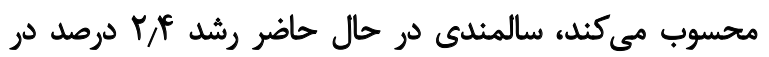

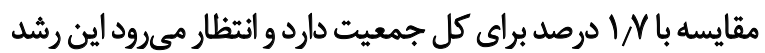

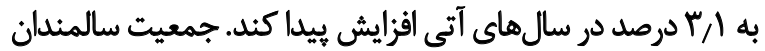

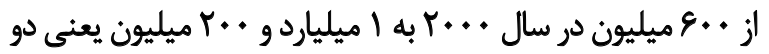

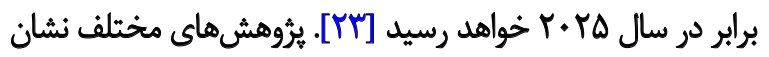

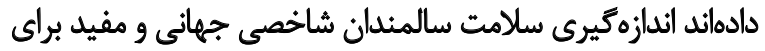

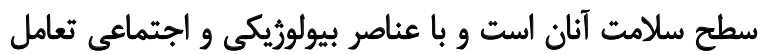

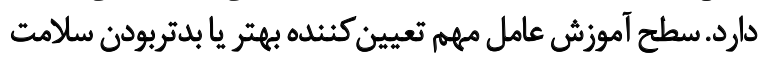

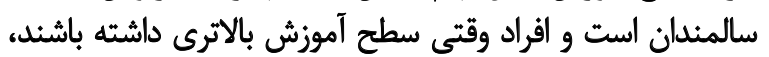

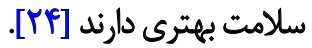

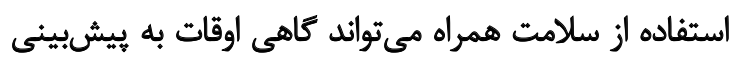

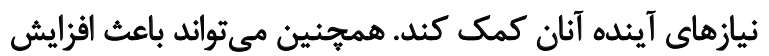

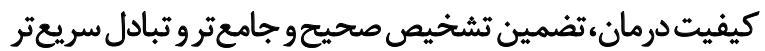

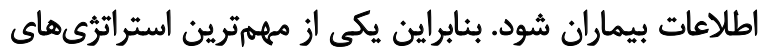

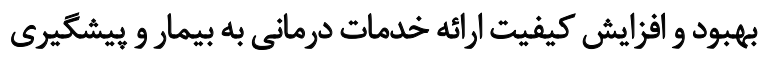
از خطاهاى يزشكى محسوب مي خئود.

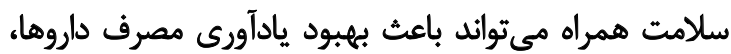
ارسال دادههاى درخواستى بيماران در هر زمان ومان ومكان و همجنين 


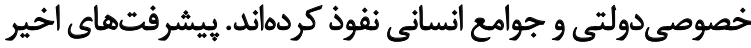

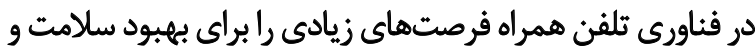

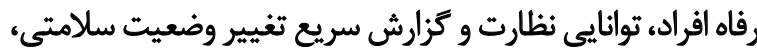

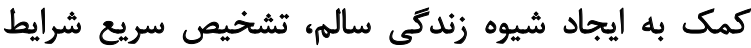

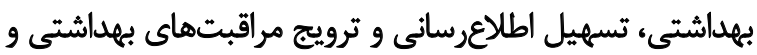

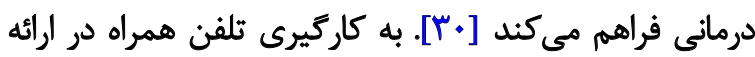

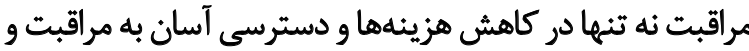

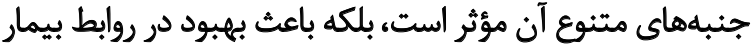

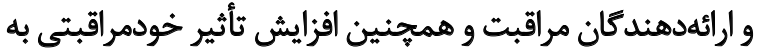

خصوص در سالمندى مى مرود [آست]

اين فناورى مىتواند در احساس استقلال و امنيت سالمندان

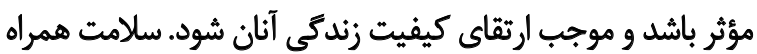

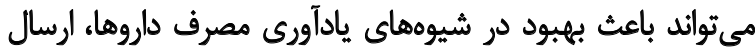

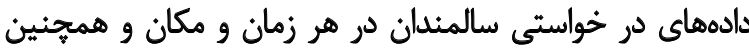

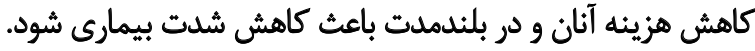

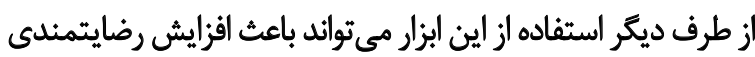

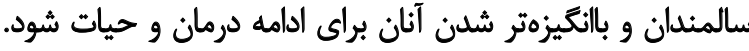

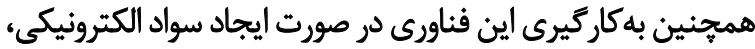

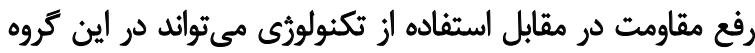

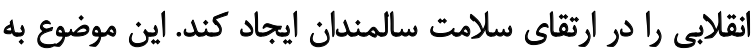
فراهمرساختن زيرساختارها، نظامنامه و استاندارد و تحقيقات بيشترى

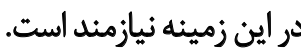

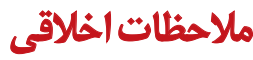

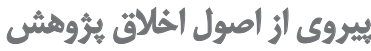

در انجام اين يثوهش، نيازى به بيروى از اصول اخلاق يُوهش نبوده است.

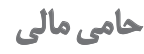

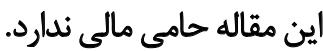

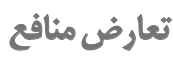

بنا به اظهار نظر نويسندكان اين مقاله تعارض مثافع ندارد. 


\section{References}

[1] Javadian SR. [Gerontological social work (Persian)]. Journal of Social Work. 2015; 4(2):27-32.

[2] Jesmani M, Sharifian R, Khalesi H. [The role of mobile wireless devices in health care and its relationship with self-care (Persian)] Paper presented at the Shiraz International Mobile Health Seminar: SIM Seminar; 17-18 May 2015; Shiraz, Iran.

[3] World Health Organization. World report on ageing and health. Geneva: World Health Organization; 2015.

[4] Taghdisi MH, Estebsari F, Rahimi Foroushani A, Eftekhar Ardebili $\mathrm{H}$, Shojaeizadeh D, Dastoorpoor M et al. [The educational program based on the successful aging approach in elders healthpromoting behaviors: A clinical trial study (Persian)]. The Razi Journal of Medical Sciences. 2014; 21(125):26-36.

[5] Riahi F, Izadi-mazidi M, khajedin N, norouzi S. [Does education of geriatric medicine effect on the Medical Students' Attitude toward Elderlies and Their Care? (Persian)]. Iranian Journal of Medical Education. 2014; 14(7):651-2.

[6] Morasae EK, Forouzan AS, Majdzadeh R, Asadi-Lari M, Noorbala AA, Hosseinpoor AR. Understanding determinants of socioeconomic inequality in mental health in Iran's capital, Tehran: A concentration index decomposition approach. International Journal for Equity in Health. 2012; 11(1):18. [DOI:10.1186/1475-927611-18] [PMID] [PMCID]

[7] Hoodfar H, Assadpour S. The politics of population policy in the Islamic Republic of Iran. Studies in Family Planning. 2000; 31(1):19-34. [DOI:10.1111/j.1728-4465.2000.00019.x] [PMID]

[8] Ajami S, Ahmadi B. [Information Technology in Healthcare Provide Process by Physicians (Persian)]. Health Information Management. 12(5):555-6.

[9] Borrel-carrio F, Suchman AL, Epsteir RM. The Biopsychosocial Model 25 Years Later: Principles, Practice, and Scientific Inquiry. Annals of Family Medicine. 2004; 2(6):576-82.

[10] Mahmoodi mehr z, Dr. Sharifian R, Fallah nejad E, Zaker S, Jelvay S. [M_health mobile health application in patients with asthma (Persian)]. Paper presented at the Shiraz International Mobile Health Seminar: SIM Seminar; 17-18 May 2015; Shiraz, Iran.

[11] Tadayyon H, Safdari R, khademi H, Sheikh_al_tayefe M. [Mobile health technology as a tool for medical diagnosis (Persian)]. Paper presented at the Shiraz International Mobile Health Seminar: SIM Seminar; 17-18 May 2015; Shiraz, Iran.

[12] Thiromurty H, Lester RT. M-health for health behavior change in resource-limited setting; applications to HIV care and beyond. Bulletin of the World Health Organization. 2012; 90(5):390-2. [DOI:10.2471/BLT.11.099317] [PMID] [PMCID]

[13] Kazemi J,Monem H,Ravangard R, Zaker S, Dr. Sharifian R :Introducing a questionnaire to measure the acceptance of patient portals laboratory using equipment. Paper presented at theShiraz International Mobile Health Seminar: SIM Seminar; 17-18 May 2015; Shiraz, Iran.

[14] Aghamohamadi T, Aghamohamadi Said Bagher maddah S, Mohammadi Shahbolaghi SF, Dalvandi A, Khaleghipour M. [The impact of self-management program on self-efficacy of elderly patients with heart failure (Persian)]. Journal of Urmia Nursing And Midwifery Faculty. 2017; 14(12):1013-23.
[15] Antonicelli R, Testarmata P, Spazzafumo L, Gagliardi C, Bilo G Valentini $\mathrm{M}$, et al. Impact of telemonitoring at home on the management of elderly patients with congestive heart failure. Journal of Telemedicine and Telecare. 2008; 14(6):300-5. [DOI:10.1258/ jtt.2008.071213]

[16] Bloomfield GS, Vedanthan R, Vasudevan L, Kithei A, Were M, Velazquez EJ. Mobile health for non-communicable diseases in Sub-Saharan Africa: a systematic review of the literature and strategic framework for research. Journal of Global Health. 2014; 10:49. [DOI:10.1186/1744-8603-10-49] [PMID] [PMCID]

[17] Eng DS, Lee JM. The promise and peril of mobile health applications for diabetes and endocrinology. Pediatric Diabetes. 2013; 14(4):231-8. [DOI:10.1111/pedi.12034] [PMID] [PMCID]

[18] Kazemi A, Dr. Sharifian R, Farshadi R. [The role of online social networks to increase the level of patient care (Persian)]. Paper presented at the Shiraz International Mobile Health Seminar: SIM Seminar; 17-18 May 2015; Shiraz, Iran.

[19] Gandapur Y, Kianoush S, Kelli HM, Misra S, Urrea B, Blaha MJ. The role of $\mathrm{m}$-health for improving medication adherence in patients with cardiovascular disease: a systematic review. Quality of Care and Clinical Outcomes. 2016; 2(4):237-44. [DOI:10.1093/ ehjqcco/qcw018.] [PMID] [PMCID]

[20] Ajami S, Heidarinia Z. [The use of mobile-health technology for monitoring the health of the elderly (Persian)]. Health Information Management. 2015; 12(4):391-2.

[21] Zare S, Farahmand F, Bakhshi M, Abdalkhani R, Jelvai S. [Effect of mobile phone help in reducing anxiety and hope in heart patients (Persian)]. Paper presented at the Shiraz International Mobile Health Seminar: SIM Seminar; 17-18 May 2015; Shiraz, Iran.

[22] Amy J Barton: The regulation of mobile health applications. BMC Medicine. 2012; 10:46. [DOI:10.1186/1741-7015-10-46] [PMID] [PMCID]

[23] Sharifirad G, Mohebbi S, Matlabi M. The relationship of physical activity in middle age and cardiovascular problems in old age in retired people in Isfahan, 2006. The Horizon of Medical Sciences. 2007; 13(2):57-63.

[24] Askaryzadeh Mahani M, Arab M, Mohammadalizade S, Haghdoost A. [Staff Nurses Knowledge of Aging Process and Their Attitude toward Elder People (Persian)]. Iran Journal of Nursing. 2008; 21(55):19-27.

[25] Khademian F, Sharifian R, Khademian Z, Jelvai S, Niknam F, Fallah nejad E. [The impact of education on diabetes control via mobile phone (Persian)]. Paper presented at the Shiraz International Mobile Health Seminar: SIM Seminar; 17-18 May 2015; Shiraz, Iran.

[26] Jelvai S, Zareei J, Shaker Zade F, Niknam F, Darabi M. [Training SMS a way to improving care for patients with diabetes type II (Persian)]. Paper presented at the Shiraz International Mobile Health Seminar: SIM Seminar; 17-18 May 2015; Shiraz, Iran.

[27] van Heerden A, Tomlinson M, Swartz L. Point of care in your pocket: A research agenda for the field of m-health. Bulletin of the World Health Organization. 2012; 90(5):393-4. [DOI: 10.2471/ BLT.11.099788.] [PMID] [PMCID]

[28] Ocampo JM. Self-rated health: Importance of use in elderly adults. Colombia Médica. 2010; 41(3):275-89. 
[29] Jamejamonline. [Elderly Mobile Phone (Persian)]. 2014 [Updated 2014 July 3]. Available from: http://jamejamonline.ir/ sara/1554592783772246142

[30] Zahmatkeshan M, Safdari R. M_health Technology, new development in health promotion. Telemedicine (Special Issue); 2014.

[31] Alizadeh Khoei M, Hoseini M, Shojaizade D, dokhte Rahimi A, Salehe Mortazavi S. [Assessing mental health status of elderly in specific age groups in Tehran metropolitan city in 2010 (Persian)] [Internet]. 2011 [Updated 2011 July 5]. Available from: http:// research.tums.ac.ir/reports/88-04-27-9874/final-2-2011-07-05-paperJournal.doc 
